\title{
Numerical study of thermoviscous effects in ultrasound-induced acoustic streaming in microchannels
}

\author{
Muller, Peter Barkholt; Bruus, Henrik
}

Published in:

Physical Review E

Link to article, DOI:

10.1103/PhysRevE.90.043016

Publication date:

2014

Document Version

Publisher's PDF, also known as Version of record

Link back to DTU Orbit

Citation $(A P A)$ :

Muller, P. B., \& Bruus, H. (2014). Numerical study of thermoviscous effects in ultrasound-induced acoustic streaming in microchannels. Physical Review E, 90(4), 043016. https://doi.org/10.1103/PhysRevE.90.043016

\section{General rights}

Copyright and moral rights for the publications made accessible in the public portal are retained by the authors and/or other copyright owners and it is a condition of accessing publications that users recognise and abide by the legal requirements associated with these rights.

- Users may download and print one copy of any publication from the public portal for the purpose of private study or research.

- You may not further distribute the material or use it for any profit-making activity or commercial gain

- You may freely distribute the URL identifying the publication in the public portal

If you believe that this document breaches copyright please contact us providing details, and we will remove access to the work immediately and investigate your claim. 


\title{
Numerical study of thermoviscous effects in ultrasound-induced acoustic streaming in microchannels
}

\author{
Peter Barkholt Muller ${ }^{*}$ and Henrik Bruus ${ }^{\dagger}$ \\ Department of Physics, Technical University of Denmark, DTU Physics Building 309, DK-2800 Kongens Lyngby, Denmark
}

(Received 21 August 2014; published 21 October 2014)

\begin{abstract}
We present a numerical study of thermoviscous effects on the acoustic streaming flow generated by an ultrasound standing-wave resonance in a long straight microfluidic channel containing a Newtonian fluid. These effects enter primarily through the temperature and density dependence of the fluid viscosity. The resulting magnitude of the streaming flow is calculated and characterized numerically, and we find that even for thin acoustic boundary layers, the channel height affects the magnitude of the streaming flow. For the special case of a sufficiently large channel height, we have successfully validated our numerics with analytical results from 2011 by Rednikov and Sadhal for a single planar wall. We analyzed the time-averaged energy transport in the system and the time-averaged second-order temperature perturbation of the fluid. Finally, we have made three main changes in our previously published numerical scheme to improve the numerical performance: (i) The time-averaged products of first-order variables in the time-averaged second-order equations have been recast as flux densities instead of as body forces. (ii) The order of the finite-element basis functions has been increased in an optimal manner. (iii) Based on the International Association for the Properties of Water and Steam (IAPWS 1995, 2008, and 2011), we provide accurate polynomial fits in temperature for all relevant thermodynamic and transport parameters of water in the temperature range from 10 to $50{ }^{\circ} \mathrm{C}$.
\end{abstract}

DOI: 10.1103/PhysRevE.90.043016

PACS number(s): 47.15.-x, 43.25.Nm, 43.25.+y, 43.35.Ud

\section{INTRODUCTION}

Ultrasound acoustophoresis has been used to handle particles of a few micrometers to tens of micrometers in microfluidic channels [1], with applications in, e.g., up-concentration of rare samples [2], cell synchronization [3], cell trapping [4], cell patterning [5], cell detachment [6], cell separation [7], and particle rotation [8]. Control and processing of submicrometer bioparticles have many applications in biomedicine and in environmental and food analysis, however acoustophoretic focusing of submicrometer particles by the primary radiation force is hindered by the drag force from the acoustic streaming flow of the suspending liquid. Consequently, there is a need for understanding the acoustic streaming and for developing tools for engineering acoustic streaming patterns that allow for acoustic handling of submicrometer particles.

The theory of acoustic streaming, driven by the timeaveraged shear stress near rigid walls in the acoustic boundary layers of a standing wave, was originally described by Lord Rayleigh [9]. It was later extended, among others, by Schlicting [10], Nyborg [11], Hamilton [12,13], and Muller et al. [14]. Recently, Rednikov and Sadhal [15] have included the temperature dependence of the dynamic viscosity and shown that this can lead to a significant increase in the magnitude of the streaming velocity. In the present work, we present a numerical study of this and related thermoviscous effects.

A major challenge in numerical modeling of acoustic streaming is the disparate length scales characterizing the bulk of the fluid and the acoustic boundary layer, the latter often

\footnotetext{
*peter.b.muller@fysik.dtu.dk

†bruus@fysik.dtu.dk
}

being several orders of magnitude smaller than the former in relevant experiments. One way to handle this problem is to determine the first-order oscillatory acoustic field without resolving the acoustic boundary layers, and from this calculate an approximate expression for the time-averaged streaming velocity at the boundary, acting as a boundary condition for the steady bulk streaming $[16,17]$. This method has the advantage of being computationally less demanding. For example, Lei et al. $[18,19]$ used it to model streaming flow in microfluidic channels in three dimensions, and they were able to qualitatively explain several experimental observations of streaming flow in microchannels and flat microfluidics chambers. Another method is the direct numerical solution of the full thermoviscous acoustic equations both in the bulk and in the thin boundary layers, demanding a fine spatial resolution close to rigid surfaces as developed by, e.g., Muller et al. [20]. They obtained a quantitative description of the physics of the thermoviscous boundary layers and the acoustic resonance. The same model was later employed in a quantitative comparison between numerics, analytics, and experiments of microparticle acoustophoresis, demonstrating good agreement [14]. In a more recent study, the numerical scheme was further used to demonstrate how simultaneous actuation of the two overlapping half-wavelength resonances of a nearly square channel can generate a single vortex streaming flow that allows for focusing of submicrometer particles, an effect demonstrated experimentally by focusing $0.5-\mu \mathrm{m}-$ diam particles and E. coli bacteria [21].

In this paper, we extend our numerical model for a rectangular microchannel [20] to include the thermoviscous effects, which were treated analytically in the special case of a single planar infinite rigid wall by Rednikov and Sadhal [15]. The extension is done by including the dependence on the oscillatory first-order temperature and density fields 
in the dynamic shear viscosity, previously taken to be constant. This has a significant influence on the shear stresses in the thermoviscous boundary layers responsible for generating the steady acoustic streaming. Furthermore, we study the steady temperature rise and energy current densities resulting from solving the time-averaged second-order energy transport equation. Finally, we improve the convergence properties of our previous numerical scheme [20] by implementing the governing equations in a source-free flux formulation and optimizing the order of the basis functions of the finite-element scheme.

\section{BASIC THEORY}

In this section, we derive the governing equations for the first- and second-order perturbations to the thermoviscous acoustic fields in a compressible Newtonian fluid. We only consider the acoustics in the fluid, and we treat the surrounding walls as ideal hard walls. Our treatment is based on textbook thermodynamics [22] and thermoviscous acoustics [23], but in a source-free flux formulation suitable for our specific numerical implementation. As water is our model fluid of choice, we carefully implement the best available experimental data for the thermodynamic and transport parameters provided by the International Association for the Properties of Water and Steam (IAPWS).

\section{A. Thermodynamics}

The independent thermodynamic variables of the compressible Newtonian fluid are taken to be the temperature $T$ and the pressure $p$ [22]. The dependent variables are the mass density $\rho$, the internal energy $\varepsilon$ per mass unit, and the entropy $s$ per mass unit. The first law of thermodynamics is usually stated with $s$ and $\rho$ as the independent variables,

$$
d \varepsilon=T d s-p d\left(\frac{1}{\rho}\right)=T d s+\frac{p}{\rho^{2}} d \rho .
$$

By a standard Legendre transformation of $\varepsilon$ to the Gibbs free energy $g$ per unit mass, $g=\varepsilon-T s+p \frac{1}{\rho}$, we obtain the first law with $T$ and $p$ as the independent variables,

$$
d g=-s d T+\frac{1}{\rho} d p .
$$

Due to their importance in thermoacoustics, we furthermore introduce the following three thermodynamics coefficients: the isobaric heat capacity $c_{p}$ per unit mass, the isobaric thermal expansion coefficient $\alpha_{p}$, and the isothermal compressibility $\kappa_{T}$, as

$$
\begin{aligned}
c_{p} & =T\left(\frac{\partial s}{\partial T}\right)_{p}, \\
\alpha_{p} & =-\frac{1}{\rho}\left(\frac{\partial \rho}{\partial T}\right)_{p}, \\
\kappa_{T} & =\frac{1}{\rho}\left(\frac{\partial \rho}{\partial p}\right)_{T} .
\end{aligned}
$$

Moreover, as a standard step toward getting rid of explicit references to the entropy, we derive from Eqs. (1b) and (2b) the following Maxwell relation:

$$
\left(\frac{\partial s}{\partial p}\right)_{T}=-\frac{\partial^{2} g}{\partial p \partial T}=-\left(\frac{\partial\left(\frac{1}{\rho}\right)}{\partial T}\right)_{p}=-\frac{1}{\rho} \alpha_{p} .
$$

Using Eqs. (2a)-(2d), we express $d s$ and $d \rho$ in terms of $d T$ and $d p$,

$$
\begin{aligned}
T d s & =c_{p} d T-\frac{\alpha_{p} T}{\rho} d p, \\
\frac{1}{\rho} d \rho & =\kappa_{T} d p-\alpha_{p} d T,
\end{aligned}
$$

which combined with Eq. (1a) leads to $d \varepsilon$ in terms of $d T$ and $d p$,

$$
\rho d \varepsilon=\left(c_{p} \rho-\alpha_{p} p\right) d T+\left(\kappa_{T} p-\alpha_{p} T\right) d p .
$$

Using Eqs. (3a)-(3c), small changes $d s, d \rho$, and $d \varepsilon$ in the dependent thermodynamic variables $s, \rho$, and $\varepsilon$ away from equilibrium can thus be expressed in terms of changes in the independent thermodynamic variables $T$ and $p$. In our numerical analysis, the default unperturbed equilibrium state is the one at ambient temperature $T_{0}=25.0^{\circ} \mathrm{C}$ and pressure $p_{0}=0.1013 \mathrm{MPa}$.

\section{B. Physical properties of water according to IAPWS}

The theoretical treatment of thermoviscous acoustics requires detailed knowledge of the dependence on temperature and density (or temperature and pressure) of the physical properties of the fluid of choice. In the present paper, we use the parameter values for water supplied by the IAPWS in its thorough statistical treatment of large data sets provided by numerous experimental groups [24-26].

The values of the thermodynamic properties are taken from the IAPWS Formulation 1995 [24], the shear viscosity is taken from the IAPWS Formulation 2008 [25], the thermal conductivity is taken from the IAPWS Formulation 2011 [26], while the bulk viscosity is taken from Holmes, Parker, and Povey [27], who extended the work by Dukhin and Goetz [28]. The IAPWS data set spans a much wider range in temperature and ambient pressures than needed in our work, and it is somewhat complicated to handle. Consequently, to ease the access to the IAPWS data in our numerical implementation, we have carefully fitted the temperature dependence of all properties at atmospheric pressure by fifth-order polynomials in temperature in the range from 10 to $50{ }^{\circ} \mathrm{C}$, as described in detail in Appendix $\mathrm{A}$. In the specified range, the differences between our fits and the IAPWS data are negligible. In Table I, we have listed the physical properties of water at ambient temperature and pressure.

The thermodynamic coefficients of Eq. (3) are by definition evaluated at the equilibrium state $T=T_{0}$ and $p=p_{0}$, leaving all acoustics perturbations to enter only in the small deviations, e.g., $d T=T_{1}+T_{2}$. On the other hand, the transport coefficients of the fluid depend on the acoustic perturbation. To avoid the ambiguity of the pressure $p$ as either the ambient pressure outside the fluid or the intrinsic pressure (cohesive energy) of the fluid, we use Eq. (3b) to change the variable from pressure $p$ to density $\rho$ in our treatment of the IAPWS 
TABLE I. IAPWS parameter values for pure water at ambient temperature $25^{\circ} \mathrm{C}$ and pressure $0.1013 \mathrm{MPa}$. For references, see Appendix A.

\begin{tabular}{|c|c|c|c|}
\hline Parameter & Symbol & Value & Unit \\
\hline \multicolumn{4}{|c|}{ Thermodynamic parameters: } \\
\hline Mass density & $\rho$ & $9.970 \times 10^{2}$ & $\mathrm{~kg} \mathrm{~m}^{-3}$ \\
\hline Heat capacity & $c_{p}$ & $4.181 \times 10^{3}$ & $\mathrm{~J} \mathrm{~kg}^{-1} \mathrm{~K}^{-1}$ \\
\hline Speed of sound & $c_{s}^{p}$ & $1.497 \times 10^{3}$ & $\mathrm{~m} \mathrm{~s}^{-1}$ \\
\hline Compressibility & $\kappa_{T}^{s}$ & $4.525 \times 10^{-10}$ & $\mathrm{~Pa}^{-1}$ \\
\hline Thermal expansion & $\alpha_{p}$ & $2.573 \times 10^{-4}$ & $\mathrm{~K}^{-1}$ \\
\hline Heat capacity ratio & $\gamma$ & $1.011 \times 10^{0}$ & \\
\hline \multicolumn{4}{|l|}{ Transport parameters: } \\
\hline Shear viscosity & $\eta$ & $8.900 \times 10^{-4}$ & $\mathrm{~Pa} \mathrm{~s}$ \\
\hline Bulk viscosity & $\eta^{\mathrm{b}}$ & $2.485 \times 10^{-3}$ & $\mathrm{~Pa} \mathrm{~s}$ \\
\hline Thermal conductivity & $k^{\text {th }}$ & $6.065 \times 10^{-1}$ & $\mathrm{~W} \mathrm{~m} \mathrm{~m}^{-1} \mathrm{~K}^{-1}$ \\
\hline \multicolumn{4}{|c|}{ Thermodynamic derivatives: } \\
\hline & $1 \partial \eta$ & \multirow{2}{*}{$-2.278 \times 10^{-2}$} & \multirow{2}{*}{$\mathrm{K}^{-1}$} \\
\hline & $\bar{\eta} \overline{\partial T}$ & & \\
\hline & $\frac{1}{\eta} \frac{\partial \eta}{\partial \rho}$ & $-3.472 \times 10^{-4}$ & $\mathrm{~kg}^{-1} \mathrm{~m}^{3}$ \\
\hline & $1 \partial \eta^{\mathrm{b}}$ & \multirow{2}{*}{$-2.584 \times 10^{-2}$} & \multirow{2}{*}{$\mathrm{K}^{-1}$} \\
\hline & $\overline{\eta^{\mathrm{b}}} \overline{\partial T}$ & & \\
\hline & $1 \partial k^{\text {th }}$ & \multirow{2}{*}{$2.697 \times 10^{-3}$} & \multirow{2}{*}{$\mathrm{K}^{-1}$} \\
\hline & $\overline{k^{\text {th }}} \overline{\partial T}$ & & \\
\hline & $1 \frac{\partial k^{\text {th }}}{\partial}$ & \multirow{2}{*}{$2.074 \times 10^{-3}$} & \multirow{2}{*}{$\mathrm{kg}^{-1} \mathrm{~m}^{3}$} \\
\hline & $\overline{k^{\text {th }}} \overline{\partial \rho}$ & & \\
\hline
\end{tabular}

data. To first order in the acoustic perturbation, we thus write the dynamic shear viscosity $\eta$, the bulk (second) viscosity $\eta^{\mathrm{b}}$, and the thermal conductivity $k^{\text {th }}$ as

$$
\begin{aligned}
\eta(T, \rho) & =\eta_{0}\left(T_{0}, \rho_{0}\right)+\eta_{1}\left(T_{0}, T_{1}, \rho_{0}, \rho_{1}\right), \\
\eta_{1} & =\left(\frac{\partial \eta}{\partial T}\right)_{T=T_{0}} T_{1}+\left(\frac{\partial \eta}{\partial \rho}\right)_{\rho=\rho_{0}} \rho_{1}, \\
\eta^{\mathrm{b}}(T, \rho) & =\eta_{0}^{\mathrm{b}}\left(T_{0}, \rho_{0}\right)+\eta_{1}^{\mathrm{b}}\left(T_{0}, T_{1}, \rho_{0}, \rho_{1}\right), \\
\eta_{1}^{\mathrm{b}} & =\left(\frac{\partial \eta^{\mathrm{b}}}{\partial T}\right)_{T=T_{0}} T_{1}+\left(\frac{\partial \eta^{\mathrm{b}}}{\partial \rho}\right)_{\rho=\rho_{0}} \rho_{1}, \\
k^{\mathrm{th}}(T, \rho) & =k_{0}^{\mathrm{th}}\left(T_{0}, \rho_{0}\right)+k_{1}^{\text {th }}\left(T_{0}, T_{1}, \rho_{0}, \rho_{1}\right), \\
k_{1}^{\mathrm{th}} & =\left(\frac{\partial k^{\mathrm{th}}}{\partial T}\right)_{T=T_{0}} T_{1}+\left(\frac{\partial k^{\mathrm{th}}}{\partial \rho}\right)_{\rho=\rho_{0}} \rho_{1} .
\end{aligned}
$$

For the acoustic amplitudes used in this model, the maximum relative perturbations, such as $\left|\eta_{1}\right| / \eta_{0}$, due to the temperature dependence of the transport coefficients, are $0.33 \%, 0.53 \%$, and $0.034 \%$ for $\eta, \eta^{\mathrm{b}}$, and $k^{\mathrm{th}}$, respectively, and the perturbations due to the density dependence are $0.37 \%$ and $0.82 \%$ for $\eta$ and $k^{\text {th }}$, respectively. We could not find any literature on the density dependence of $\eta^{\mathrm{b}}$ of water.

\section{Governing equations}

Besides the above thermodynamic relations, the governing equations of thermoviscous acoustics requires the introduction of the velocity field $\boldsymbol{v}$ of the fluid as well as the stress tensor $\boldsymbol{\sigma}$, which is given as [29]

$$
\begin{aligned}
\boldsymbol{\sigma} & =-p \mathbf{1}+\boldsymbol{\tau}, \\
\boldsymbol{\tau} & =\eta\left[\boldsymbol{\nabla} \boldsymbol{v}+(\boldsymbol{\nabla} \boldsymbol{v})^{\mathrm{T}}\right]+\left[\eta^{\mathrm{b}}-\frac{2}{3} \eta\right](\boldsymbol{\nabla} \cdot \boldsymbol{v}) \mathbf{1} .
\end{aligned}
$$

Here, $\mathbf{1}$ is the unit tensor, and the superscript " $\mathrm{T}$ " indicates tensor transposition.

Mass conservation implies that the rate of change $\partial_{t} \rho$ of the density in a test volume with surface normal vector $\boldsymbol{n}$ is given by the influx (direction $-\boldsymbol{n}$ ) of the mass current density $\rho v$. In differential form by Gauss's theorem, it is

$$
\partial_{t} \rho=\nabla \cdot[-\rho \boldsymbol{v}] .
$$

Similarly, momentum conservation implies that the rate of change $\partial_{t}(\rho \boldsymbol{v})$ of the momentum density in the same test volume is given by the stress forces $\sigma$ acting on the surface (with normal $\boldsymbol{n}$ ), and the influx (direction $-\boldsymbol{n}$ ) of the momentum current density $\rho \boldsymbol{v} v$. In differential form, neglecting body forces $f$, this becomes

$$
\partial_{t}(\rho \boldsymbol{v})=\nabla \cdot[\boldsymbol{\tau}-p \mathbf{1}-\rho \boldsymbol{v} \boldsymbol{v}] .
$$

Finally, energy conservation implies that the rate of change $\partial_{t}\left(\rho \varepsilon+\frac{1}{2} \rho v^{2}\right)$ of the energy density (internal plus kinetic) is given by the power of the stress forces $\boldsymbol{v} \cdot \boldsymbol{\sigma}$ on the surface (direction $\boldsymbol{n}$ ), and the influx (direction $-\boldsymbol{n}$ ) of both heat conduction power $-k^{\text {th }} \nabla T$ and energy current density $\left(\rho \varepsilon+\frac{1}{2} \rho v^{2}\right) v$. In differential form, neglecting heat sources in the volume, this becomes

$$
\begin{aligned}
\partial_{t}\left(\rho \varepsilon+\frac{1}{2} \rho v^{2}\right)= & \nabla \cdot[\boldsymbol{v} \cdot \boldsymbol{\tau} \\
& \left.-p \boldsymbol{v}+k^{\text {th }} \nabla T-\rho\left(\varepsilon+\frac{1}{2} v^{2}\right) \boldsymbol{v}\right] .
\end{aligned}
$$

\section{First-order equations of thermoviscous acoustics}

The homogeneous, isotropic quiescent state (thermal equilibrium) is taken to be the zeroth-order state in the acoustic perturbation expansion. Following standard first-order perturbation theory, all fields $g$ are written in the form $g=g_{0}+g_{1}$, for which $g_{0}$ is the value of the zeroth-order state and $g_{1}$ is the acoustic perturbation, which must be much smaller than $g_{0}$. In our work, $\left|g_{1}\right| / g_{0} \lesssim 10^{-3}$ [20]. We assume that the acoustic perturbations $g_{1}$ are oscillating harmonically with the angular frequency $\omega$ of the acoustic actuation,

$$
g_{1}(\boldsymbol{r}, t)=g_{1}(\boldsymbol{r}) e^{-i \omega t}, \quad \partial_{t} g_{1}=-i \omega g_{1} .
$$

For the velocity, the value of the zeroth-order state is $\boldsymbol{v}_{0}=\mathbf{0}$, and thus $\boldsymbol{v}=\boldsymbol{v}_{1}$. The zeroth-order terms solve the governing equations for the zeroth-order state and thus drop out of the equations. Keeping only first-order terms, we obtain the firstorder equations.

The continuity Eq. (6a) becomes

$$
\partial_{t} \rho_{1}=-\rho_{0} \nabla \cdot \boldsymbol{v}_{1}
$$

which, by using Eq. (3b) in the form

$$
\rho_{1}=\rho_{0}\left[\kappa_{T} p_{1}-\alpha_{p} T_{1}\right]
$$

is rewritten as

$$
\alpha_{p} \partial_{t} T_{1}-\kappa_{T} \partial_{t} p_{1}=\nabla \cdot v_{1}
$$


The momentum Eq. (6b) likewise becomes

$$
\rho_{0} \partial_{t} \boldsymbol{v}_{1}=\nabla \cdot\left[\boldsymbol{\tau}_{1}-p_{1} \mathbf{1}\right],
$$

where $\tau_{1}$ is given by

$$
\boldsymbol{\tau}_{1}=\eta_{0}\left[\nabla \boldsymbol{v}_{1}+\left(\nabla \boldsymbol{v}_{1}\right)^{\mathrm{T}}\right]+\left[\eta_{0}^{\mathrm{b}}-\frac{2}{3} \eta_{0}\right]\left(\boldsymbol{\nabla} \cdot \boldsymbol{v}_{1}\right) \mathbf{1}
$$

The energy Eq. (6c) requires a little more work. To begin with, it can be written as

$$
\rho_{0} \partial_{t} \varepsilon_{1}+\varepsilon_{0} \partial_{t} \rho_{1}=k_{0}^{\text {th }} \nabla^{2} T_{1}-p_{0} \nabla \cdot v_{1}-\varepsilon_{0} \rho_{0} \nabla \cdot v_{1} .
$$

The two terms containing $\varepsilon_{0}$ cancel out due to the continuity Eq. (8a), and the term $\rho_{0} \partial_{t} \varepsilon_{1}$ is rewritten using Eq. (1a), whereby

$$
\rho_{0} T_{0} \partial_{t} s_{1}+\frac{p_{0}}{\rho_{0}} \partial_{t} \rho_{1}=k_{0}^{\mathrm{th}} \nabla^{2} T_{1}-p_{0} \nabla \cdot v_{1} .
$$

The two terms containing $p_{0}$ cancel out due to the continuity Eq. (8a), and the term $\rho_{0} T_{0} \partial_{t} s_{1}$ is rewritten using the time derivative of Eq. (3a). This leads to

$$
\rho_{0} c_{p} \partial_{t} T_{1}-\alpha_{p} T_{0} \partial_{t} p_{1}=k_{0}^{\mathrm{th}} \nabla^{2} T_{1} .
$$

Equations (8c), (9a), and (10c) are the resulting firstorder thermoviscous equations for conservation of mass, momentum, and energy, respectively. In the frequency domain, they become

$$
\begin{aligned}
-i \omega \alpha_{p} T_{1}+i \omega \kappa_{T} p_{1} & =\nabla \cdot \boldsymbol{v}_{1}, \\
-i \omega \rho_{0} \boldsymbol{v}_{1} & =\nabla \cdot\left[\boldsymbol{\tau}_{1}-p_{1} \mathbf{1}\right], \\
-i \omega \rho_{0} c_{p} T_{1}+i \omega \alpha_{p} T_{0} p_{1} & =k_{0}^{\mathrm{th}} \nabla^{2} T_{1} .
\end{aligned}
$$

From Eqs. (11b) and (11c), neglecting the pressure terms, we can derive the length scales $\delta_{\mathrm{s}}$ and $\delta_{\mathrm{t}}$ for diffusion of momentum and heat, respectively,

$$
\begin{aligned}
& \delta_{\mathrm{s}}=\sqrt{\frac{2 \eta_{0}}{\rho_{0} \omega}}=\sqrt{\frac{2 v}{\omega}}=0.38 \mu \mathrm{m}, \\
& \delta_{\mathrm{t}}=\sqrt{\frac{2 k_{0}^{\mathrm{th}}}{\rho_{0} c_{p} \omega}}=\sqrt{\frac{2 D_{\mathrm{th}}}{\omega}}=0.15 \mu \mathrm{m},
\end{aligned}
$$

where the subscript "s" indicates shear stress, subscript " $\mathrm{t}$ " indicates thermal, and $v=\eta_{0} / \rho_{0}$ and $D_{\text {th }}=k^{\text {th }} /\left(\rho_{0} c_{p}\right)$ are the momentum and thermal diffusivities with numerical values derived from the parameter values at ambient temperature and pressure listed in Table I.

\section{E. Second-order time-averaged equations of thermoviscous acoustics}

Moving on to second-order perturbation theory, writing the fields as $g=g_{0}+g_{1}+g_{2}$, we note that the second-order acoustic perturbation $g_{2}$ may contain both oscillating terms and a time-constant term. The time averaging over one oscillation period of a field $g(t)$ is denoted $\langle g\rangle$. We note that all full time derivatives average to zero, $\left\langle\partial_{t} g(t)\right\rangle=0$, and that in our work $\left|g_{2}\right| /\left|g_{1}\right| \lesssim 10^{-3}$.

In the following, all pure second-order fields are taken to be time-averaged and thus written plainly as $g_{2}$ without the angled brackets. With this notation, the second-order time-averaged continuity Eq. (6a) becomes

$$
\boldsymbol{\nabla} \cdot\left[\rho_{0} \boldsymbol{v}_{2}+\left\langle\rho_{1} \boldsymbol{v}_{1}\right\rangle\right]=0
$$

while the momentum Eq. (6b) takes the form

$$
\boldsymbol{\nabla} \cdot\left[\boldsymbol{\tau}_{2}-p_{2} \mathbf{1}-\rho_{0}\left\langle\boldsymbol{v}_{1} \boldsymbol{v}_{1}\right\rangle\right]=\mathbf{0},
$$

where $\boldsymbol{\tau}_{2}$ is given by

$$
\begin{aligned}
\boldsymbol{\tau}_{2}= & \eta_{0}\left[\boldsymbol{\nabla} \boldsymbol{v}_{2}+\left(\boldsymbol{\nabla} \boldsymbol{v}_{2}\right)^{\mathrm{T}}\right]+\left[\eta_{0}^{\mathrm{b}}-\frac{2}{3} \eta_{0}\right]\left(\boldsymbol{\nabla} \cdot \boldsymbol{v}_{2}\right) \mathbf{1} \\
& +\left\langle\eta_{1}\left[\nabla \boldsymbol{v}_{1}+\left(\nabla \boldsymbol{v}_{1}\right)^{\mathrm{T}}\right]\right\rangle+\left\langle\left[\eta_{1}^{\mathrm{b}}-\frac{2}{3} \eta_{1}\right]\left(\boldsymbol{\nabla} \cdot \boldsymbol{v}_{1}\right) \mathbf{1}\right\rangle
\end{aligned}
$$

It is in the two last terms that the first-order temperature and density dependence of the viscosities come into play through the perturbations $\eta_{1}$ and $\eta_{1}^{\mathrm{b}}$. Note that the secondorder temperature perturbation does not enter the governing equations for $\boldsymbol{v}_{2}$. Note also that both shear-induced (Rayleigh) streaming and bulk-absorption-induced Eckart streaming are included through the shear and bulk viscosity $\eta$ and $\eta^{\mathrm{b}}$, respectively.

The energy Eq. (6c) in its second-order time-averaged form is initially written as

$$
\begin{aligned}
\nabla \cdot[ & \left\langle\boldsymbol{v}_{1} \cdot \boldsymbol{\tau}_{1}\right\rangle+k_{0}^{\text {th }} \nabla T_{2}+\left\langle k_{1}^{\text {th }} \nabla T_{1}\right\rangle-p_{0} \boldsymbol{v}_{2} \\
& \left.-\left\langle p_{1} \boldsymbol{v}_{1}\right\rangle-\varepsilon_{0} \rho_{0} \boldsymbol{v}_{2}-\varepsilon_{0}\left\langle\rho_{1} \boldsymbol{v}_{1}\right\rangle-\rho_{0}\left\langle\varepsilon_{1} \boldsymbol{v}_{1}\right\rangle\right]=0 .
\end{aligned}
$$

The two terms with $\varepsilon_{0}$ cancel due to the continuity Eq. (13). Next, using Eq. (1a), we obtain the expression $\rho_{0} \varepsilon_{1}=$ $\rho_{0} T_{0} s_{1}+\left(p_{0} / \rho_{0}\right) \rho_{1}$, which upon insertion into Eq. (15a) leads to

$$
\begin{aligned}
\nabla \cdot[ & \left\langle\boldsymbol{v}_{1} \cdot \boldsymbol{\tau}_{1}\right\rangle+k_{0}^{\text {th }} \nabla T_{2}+\left\langle k_{1}^{\text {th }} \nabla T_{1}\right\rangle-p_{0} \boldsymbol{v}_{2} \\
& \left.-\left\langle p_{1} \boldsymbol{v}_{1}\right\rangle-\rho_{0} T_{0}\left\langle s_{1} \boldsymbol{v}_{1}\right\rangle-\frac{p_{0}}{\rho_{0}}\left\langle\rho_{1} \boldsymbol{v}_{1}\right\rangle\right]=0 .
\end{aligned}
$$

The two $p_{0}$ terms cancel by the continuity Eq. (13). Then, from Eq. (3a) we find $\rho_{0} T_{0} s_{1}=\rho_{0} c_{p} T_{1}-\alpha_{p} T_{0} p_{1}$, which by substitution into Eq. (15b) yields

$$
\begin{aligned}
& \boldsymbol{\nabla} \cdot\left[k_{0}^{\mathrm{th}} \nabla T_{2}+\left\langle k_{1}^{\mathrm{th}} \nabla T_{1}\right\rangle+\left\langle\boldsymbol{v}_{1} \cdot \boldsymbol{\tau}_{1}\right\rangle\right. \\
& \left.\quad-\left(1-\alpha_{p} T_{0}\right)\left\langle p_{1} \boldsymbol{v}_{1}\right\rangle-\rho_{0} c_{p}\left\langle T_{1} \boldsymbol{v}_{1}\right\rangle\right]=0 .
\end{aligned}
$$

Equations (13), (14a), and (15c) are the resulting timeaveraged second-order thermoviscous acoustic equations for conservation of mass, momentum, and energy, respectively.

The time-averaged acoustic energy density $E_{\mathrm{ac}}$ in the fluid is given by [23]

$$
E_{\mathrm{ac}}=\frac{1}{2} \kappa_{s}\left\langle p_{1}^{2}\right\rangle+\frac{1}{2} \rho_{0}\left\langle v_{1}^{2}\right\rangle,
$$

where $\kappa_{s}=\kappa_{T} / \gamma$ is the isentropic compressibility and $\gamma=$ $c_{p} / c_{V}$ is the ratio of specific heat capacities.

For a product of two time-harmonic fields in the complexvalued representation Eq. (7), the time average can be 


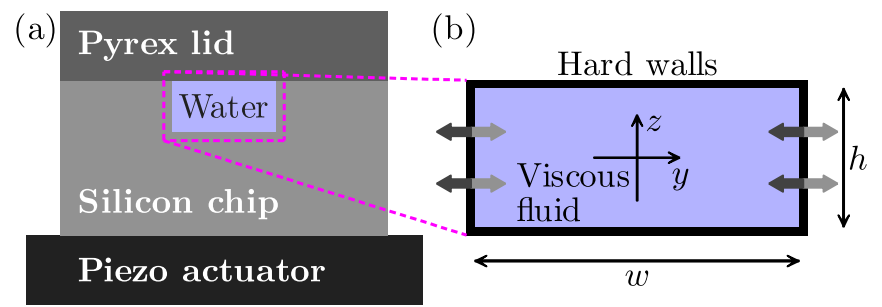

FIG. 1. (Color online) (a) Sketch of a physical acoustophoresis setup with a silicon microfluidic chip on top of a piezoactuator such as in Ref. [30]. (b) Sketch of the model system for the numerical scheme with the viscous fluid domain surrounded by hard walls. The thick arrows indicate in-phase oscillating displacement of the left and right walls. By default, we set the width $w=380 \mu \mathrm{m}$ and the height $h=160 \mu \mathrm{m}$ as in Ref. [30].

calculated as

$$
\left\langle\rho_{1}(\boldsymbol{r}, t) \boldsymbol{v}_{1}(\boldsymbol{r}, t)\right\rangle=\frac{1}{2} \operatorname{Re}\left[\rho_{1}(\boldsymbol{r}, 0) \boldsymbol{v}_{1}^{*}(\boldsymbol{r}, 0)\right],
$$

where the asterisk denote complex conjugation.

\section{NUMERICAL MODEL}

The numerical scheme solves the governing equations for the acoustic field inside the two-dimensional water domain of a rectangular microchannel cross section, whereas the vibrations in the surrounding chip material and piezotransducer are not modeled. The water domain is surrounded by immovable hard walls, and the acoustic field is excited by oscillating velocity boundary conditions, representing an oscillating nm-sized displacement of the walls. A sketch of the physical system and the numerical model is shown in Fig. 1.

\section{A. Governing equations}

The governing equations are solved using the commercial software COMSOL MULTIPHYSICS [31]. To achieve greater flexibility, the equations are implemented through mathematics-weak-form-PDE modules and not through the built-in modules for acoustics and fluid mechanics. In contrast to our previous work [20], the second-order Eqs. (13), (14a), and $(15 \mathrm{c})$ are implemented in the flux density formulation, which by partial integration avoids the less accurate secondorder derivatives appearing in the body-force formulation. To fix the numerical solution for the second-order mass and momentum conservation equations, the spatial average of the second-order pressure is forced to be zero by a Lagrange multiplier.

\section{B. Boundary conditions}

The first-order acoustic fields are solved in the frequency domain for a driven system, in which energy is added to the system by an oscillating velocity boundary condition and lost by thermal conduction through the walls. The walls are modeled as hard thermal conductors with infinite acoustic impedance and infinite thermal diffusivity. This approximation is reasonable given the parameter values listed in Table II. In the numerical model, this is implemented by zero velocity and
TABLE II. Acoustic impedance and thermal diffusivity for water, silicon, and Pyrex glass at room-temperature values from Ref. [29].

\begin{tabular}{lcc}
\hline \hline Material & $\begin{array}{c}\text { Acoustic impedance } \\
\left(10^{6} \mathrm{~kg} \mathrm{~m}^{-2} \mathrm{~s}^{-1}\right)\end{array}$ & $\begin{array}{c}\text { Thermal diffusivity } \\
\left(10^{-7} \mathrm{~m}^{2} \mathrm{~s}^{-1}\right)\end{array}$ \\
\hline Water & 1.5 & 1.4 \\
Silicon & 20 & 920 \\
Pyrex glass & 17 & 6.3 \\
\hline \hline
\end{tabular}

ambient temperature at the walls,

$$
\begin{aligned}
T & =T_{0} \quad \text { on all walls, } \\
\boldsymbol{v} & =\mathbf{0} \quad \text { on all walls, } \\
\boldsymbol{n} \cdot \boldsymbol{v}_{1} & =v_{\mathrm{bc}}(y, z) e^{-i \omega t} \text { added to actuated walls, } \\
\boldsymbol{n} \cdot \boldsymbol{v}_{2} & =-\left\langle\frac{\rho_{1}}{\rho_{0}}\left(\boldsymbol{n} \cdot \boldsymbol{v}_{1}\right)\right\rangle \text { added to actuated walls. }
\end{aligned}
$$

Here, $\boldsymbol{n}$ is the outward pointing surface normal, and Eq. (18d) ensures zero mass flux across the boundary. With the fixed temperature at the channel boundaries, we focus on the thermal properties of the acoustic effects, and we do not model any effects of heat generated by the piezotransducer. In the related experiments, the chip and the piezotransducer are thermally stabilized by a Peltier cooling element beneath the piezotransducer, which keeps the chip at a constant temperature using a temperature sensor and a PID controller; see Ref. [30] for a detailed explanation.

It is not trivial how to apply the oscillating velocity boundary condition. In our model, we wish to excite the horizontal half-wavelength resonance, which at the top and bottom walls leads to viscous boundary layers and the generation of streaming flow. To avoid direct influence on this flow from the actuation, we therefore choose to actuate only the left and right walls at $y= \pm w / 2$. Moreover, an optimal coupling to the half-wavelength resonance is obtained by choosing the proper symmetry of the actuation, and therefore in terms of the components $v_{y 1}$ and $v_{z 1}$, the boundary condition on $v_{1}$ becomes

$$
v_{y 1}\left( \pm \frac{w}{2}, z\right)=v_{\mathrm{bc}} e^{-i \omega t}, \quad v_{z 1}\left( \pm \frac{w}{2}, z\right)=0
$$

where $v_{\mathrm{bc}}=\omega d$ is the amplitude of the actuation in terms of the displacement $d$, with $d=0.1 \mathrm{~nm}$ in all simulations. This velocity boundary condition is well defined and yields results consistent with experiments [14].

\section{Convergence analysis}

The weak form equations along with the boundary conditions are solved on a two-dimensional triangular mesh using the finite-element method; see Fig. 2. The resolution of the physical field is determined by the spatial resolution of the mesh and the polynomial order of the basis functions used to represent the field in each node in the mesh. To test the validity of the numerical model, we first check that the numerical solution has converged, i.e., ensuring that further refining of the mesh does not change the solution significantly. 
(a)
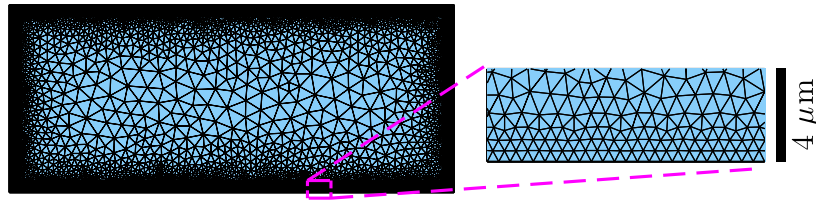

(b)
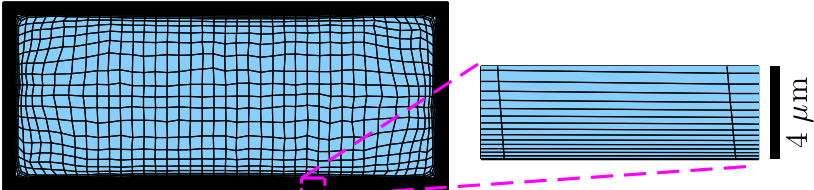

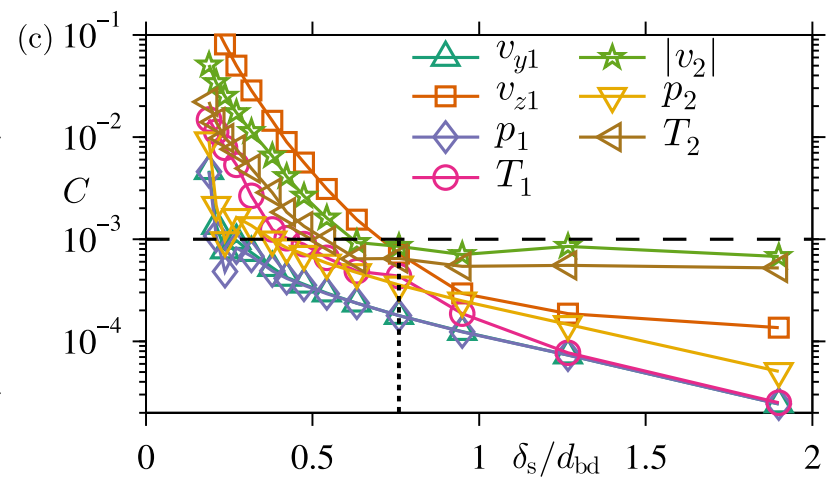

FIG. 2. (Color online) (a) The used triangular mesh with a gradually increasing element size from $0.5 \mu \mathrm{m}$ at the boundaries to $20 \mu \mathrm{m}$ in the bulk. This mesh, chosen as the default mesh, contains 30246 elements. (b) Rectangular mesh with thin elongated $0.1-\mu \mathrm{m}-\mathrm{by}-10-\mu \mathrm{m}$ elements at the boundaries and gradually changing to nearly square $10-\mu \mathrm{m}$-by-10- $\mu \mathrm{m}$ elements in the bulk. This mesh contains 3308 elements. (c) The convergence parameter $C$ of Eq. (20) for all first- and second-order fields vs the numerical resolution defined by $\delta_{\mathrm{s}} / d_{\mathrm{bd}}$, where $d_{\mathrm{bd}}$ is the mesh-element size at the boundary. The fields are solved on triangular meshes with different boundary element sizes but all with fixed bulk element size $d_{\mathrm{bk}}=20 \mu \mathrm{m}$ and growth rate $\alpha=1.3$, while the reference solution is calculated for $d_{\mathrm{bd}}=0.15 \mu \mathrm{m}, d_{\mathrm{bk}}=2 \mu \mathrm{m}$, and $\alpha=1.3$. The vertical dotted line indicates the solution for $d_{\mathrm{bd}}=0.5 \mu \mathrm{m}$, which is chosen as the default value for the following simulations.

Due to the very different length scales of the channel dimensions and the boundary layer thickness an inhomogeneous mesh is necessary, and thus there is a number of ways to refine the mesh. We used three parameters: maximum mesh-element size at the boundaries $d_{\mathrm{bd}}$, maximum mesh-element size in the bulk $d_{\mathrm{bk}}$, and the maximum mesh-element growth rate $\alpha$ (maximum relative size of neighboring elements). The convergence of the fields was considered through the relative convergence parameter $C(g)$ defined in Ref. [20] by

$$
C(g)=\sqrt{\frac{\int\left(g-g_{\mathrm{ref}}\right)^{2} d y d z}{\int\left(g_{\mathrm{ref}}\right)^{2} d y d z}},
$$

where $C(g)$ is the relative convergence of a solution $g$ with respect to a reference solution $g_{\text {ref }}$. Convergence graphs for all fields as a function of $d_{\mathrm{bd}}$ are shown in Fig. 2(c). The mesh parameters for the reference solution are $d_{\mathrm{bd}}=0.15 \mu \mathrm{m}$, $d_{\mathrm{bk}}=2 \mu \mathrm{m}$, and $\alpha=1.3$, whereas other solutions for given $d_{\mathrm{bd}}$ use $d_{\mathrm{bk}}=20 \mu \mathrm{m}$ and $\alpha=1.3$. The basis functions for the first- and second-order velocity and temperature fields are all fourth order, while for the first- and second-order pressure they are third order. All fields exhibit good convergence, and we choose $C=10^{-3}$ as our convergence criterion in the following. The corresponding default triangular mesh has $d_{\mathrm{bd}}=0.5 \mu \mathrm{m}$; see Fig. 2(a). In Fig. 2(b), a mesh is shown with rectangular mesh elements that are nearly square in the bulk of the channel while very elongated near the walls. This mesh has been used for testing purposes as it contains approximately ten times fewer mesh elements compared to the default triangular mesh, and the resulting fields all show convergence parameters below $C=10^{-3}$ with respect to the triangular reference mesh. All results have been calculated using the triangular mesh, but the square mesh provides a huge advantage regarding calculation speed and memory requirement.

\section{RESULTS}

\section{A. Resonance analysis}

To determine the acoustic resonance frequency $f_{\text {res }}$ corresponding to the horizontal half-wavelength resonance, we sweep the actuation frequency around the ideal frequency $f_{0}=$ $c_{s} / 2 w$, corresponding to the half-wavelength match $\lambda / 2=w$, and we calculate the acoustic energy density Eq. (16), shown in Fig. 3. The resonance frequency $f_{\text {res }}$ is shifted slightly with respect to the ideal frequency $f_{0}$ due to the viscous loss in the boundary layers. This loss also determines the width of the resonance curve and thus the $Q$ value of the acoustic cavity.

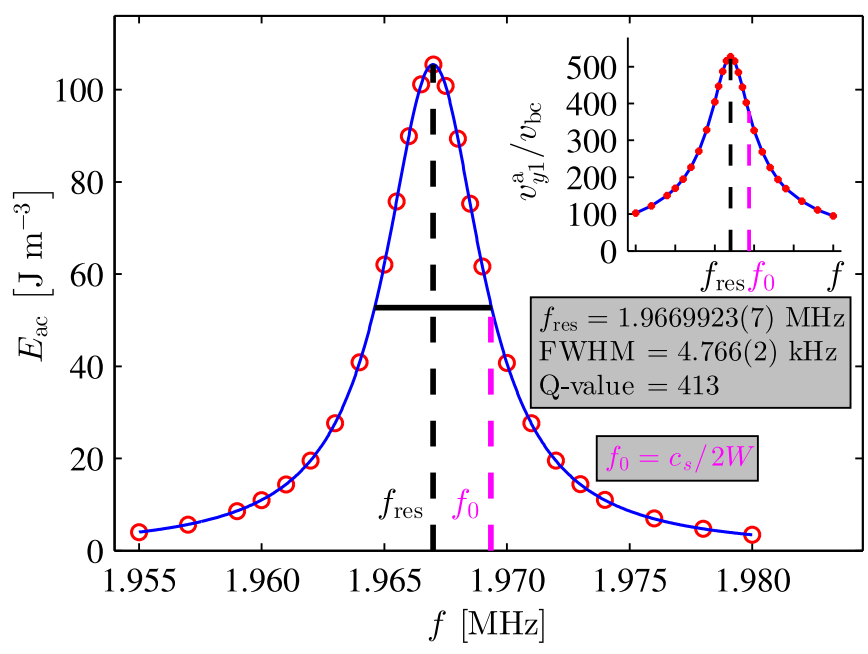

FIG. 3. (Color online) Graph of the acoustic energy density $E_{\text {ac }}$ Eq. (16) as a function of the frequency $f$ of the oscillating boundary condition. $f_{\text {res }}$ is the resonance frequency at the center of the peak, while $f_{0}$ is the ideal frequency corresponding to matching a halfwavelength with the channel width. The inset shows the magnitude of the resonant oscillating first-order velocity field $v_{y 1}^{\mathrm{a}}$ relative to the amplitude of the oscillating velocity boundary condition $v_{\mathrm{bc}}$ as a function of the actuation frequency $f$. 

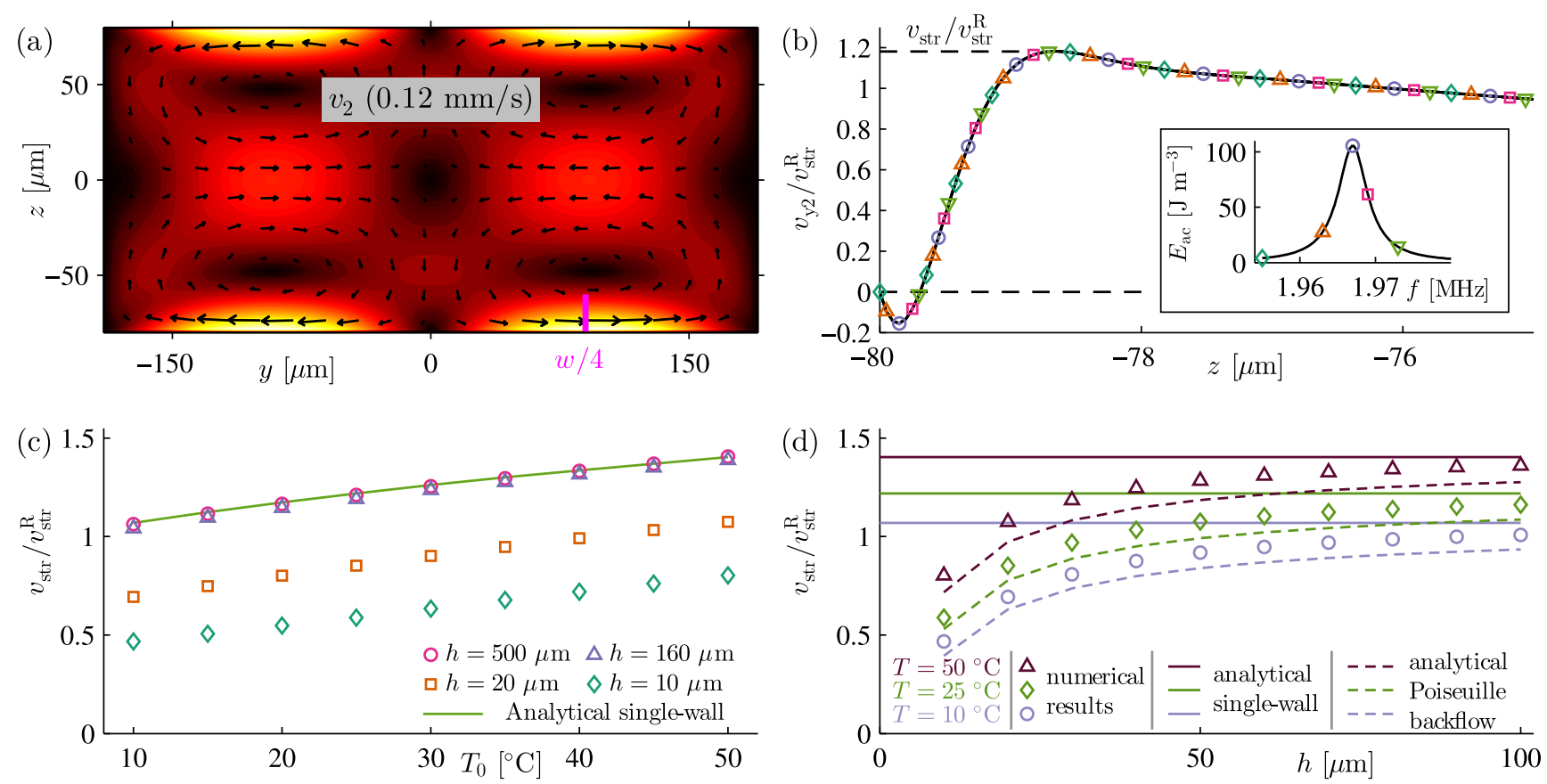

FIG. 4. (Color online) (a) Time-averaged second-order fluid velocity field $\boldsymbol{v}_{2}$ (vectors) and its magnitude [color plot ranging from $0 \mathrm{~mm} / \mathrm{s}$ (black) to $0.13 \mathrm{~mm} / \mathrm{s}$ (white)] in the vertical channel cross section calculated at $T_{0}=25{ }^{\circ} \mathrm{C}$ and $f_{\text {res }}=1.9669923 \mathrm{MHz}$. (b) The horizontal velocity component $v_{y 2}$ plotted along $y=w / 4$ indicated by the magenta line in (a). The velocity field has been calculated for the five actuation frequencies shown in the inset resonance curve, and normalized to the analytical Rayleigh streaming magnitude $v_{\mathrm{str}}^{\mathrm{R}}=(3 / 8)\left(v_{y 1}^{\mathrm{a}}\right)^{2} / c_{s}$, which is calculated based on the corresponding first-order solutions. The symbols are plotted in selected points illustrating the five numerical solutions (black lines) that coincide. (c) Normalized streaming magnitude $v_{\text {str }} / v_{\text {str }}^{\mathrm{R}}$ (symbols) vs equilibrium temperature $T_{0}$, calculated for different channel heights $h$. The full curve shows the analytical single-wall result by Ref. [15]. (d) Normalized streaming magnitude $v_{\text {str }} / v_{\text {str }}^{\mathrm{R}}$ (symbols) vs channel height $h$, calculated for different equilibrium temperatures $T_{0}$. The full curves show the analytical single-wall result by Ref. [15], while the dashed lines show the results of a one-dimensional analytical model with a Poiseuille backflow; see Eq. (21).

\section{B. Time-averaged second-order velocity}

The time-averaged second-order velocity field $\boldsymbol{v}_{2}$ is shown in Fig. 4(a), calculated for the default 380- $\mu \mathrm{m}-\mathrm{by}-160-\mu \mathrm{m}$ rectangular geometry, at $T_{0}=25^{\circ} \mathrm{C}$, and at the resonance frequency $f_{\text {res }}=1.9669923 \mathrm{MHz}$. It exhibits the well-known pattern of four flow rolls each $\lambda / 4$ wide. To investigate the magnitude of the streaming velocity, Fig. 4(b) shows the velocity along a line perpendicular to the bottom wall at $y=w / 4$. The streaming velocity field has been calculated for five different frequencies shown in the inset resonance curve. The streaming velocities have been normalized to the classical result by Lord Rayleigh for the magnitude of the acoustic streaming $v_{\mathrm{str}}^{\mathrm{R}}=(3 / 8)\left(v_{y 1}^{\mathrm{a}}\right)^{2} / c_{s}$, where $v_{y 1}^{\mathrm{a}}$ is taken from the corresponding first-order solutions. The five numerical solutions (black lines) coincide completely, showing that the rescaled second-order velocity field is the same for off-resonance actuation frequencies. This is important for our further analysis, as we do not need to determine the exact resonance frequency as it changes due to variations in temperature $T_{0}$ and channel height $h$. The magnitude of the streaming velocity $v_{\text {str }}$ is determined by the maximum value of $v_{y 2}$ along the line $y=w / 4$ as indicated in Fig. 4(b).

In Fig. 4(c), we show the normalized magnitude of the streaming velocity $v_{\text {str }} / v_{\text {str }}^{\mathrm{R}}$ versus the equilibrium temperature $T_{0}$. The streaming velocity has been calculated for different channel heights indicated by different colors and symbols. The full line is the analytical single-wall solution by Rednikov and Sadhal [15] for a standing acoustic wave parallel to a single planar wall. For all channel heights, the streaming velocity shows an almost linear dependence with positive slope on the equilibrium temperature. The numerical results for the tall channel $h=500 \mu \mathrm{m}$ agree well with the analytical single-wall prediction, while for more shallow channels the streaming velocity is significantly lower. At $25^{\circ} \mathrm{C}$, the streaming velocity is $19 \%$ larger than the classical Rayleigh result, while for $50{ }^{\circ} \mathrm{C}$ this deviation has increased to $39 \%$.

To elaborate on the dependence of the streaming velocity on the height of the channel, we plot in Fig. 4(d) the normalized streaming velocity versus the channel height for three equilibrium temperatures. The numerical results are shown by symbols, while the analytical single-wall predictions for each temperature are shown by full lines. The numerical results for the rectangular channel deviate from the analytical single-wall prediction as the channel height is decreased. To qualitatively explain this deviation, we make a simple one-dimensional analytical model along the $z$ dimension of the rectangular channel in which we impose a boundary-driven flow. The first part of the model is a plug flow with an exponential dependence close to the wall, $v_{\text {plug }}(z)=v_{0}\left\{1-\exp \left[-(z+h / 2) / \delta_{\mathrm{s}}\right]\right\}$ for $-h / 2<z<0$. This approximates the $z$ dependence of the streaming velocity field inside the viscous boundary layer, where $v_{0}$ corresponds to the analytical single-wall solution [15]. As the water is pushed toward the sidewall, a pressure 
builds up and a Poiseuille backflow is established, which by mass conservation and no-slip boundary conditions becomes $v_{\text {poi }}(z) \approx-v_{0}\left\{6\left(1 / 4-z^{2} / h^{2}\right)\right\}$. By a first-order Taylor expansion of $v_{\text {poi }}(z)$ at the wall $z=-h / 2$, we can determine the maximum $v_{\text {str }}$ of $v_{\text {plug }}(z)+v_{\text {poi }}(z)$ near the wall to first order in $\delta_{\mathrm{s}} / h$,

$$
v_{\mathrm{str}} \approx v_{0}\left\{1-6 \frac{\delta_{\mathrm{s}}}{h}\left[1+\ln \left(\frac{h}{6 \delta_{\mathrm{s}}}\right)\right]\right\} .
$$

This provides an estimate for the magnitude of the acoustic streaming shown by dashed lines in Fig. 4(d), with the viscous boundary-layer thickness Eq. (12a) calculated for each of the three temperatures. This simple one-dimensional analytical model captures the trend of the numerical data well, though overall it predicts slightly lower streaming amplitudes. The deviation from the numerical data is ascribed primarily to the monotonic approximation $v_{\text {plug }}(z)$ of the $z$ dependence of the velocity inside the viscous boundary layer. The full $z$ dependence of the streaming velocity inside the viscous boundary layer is nonmonotonic and overshoots slightly before leveling. This can be seen in Fig. 4(b), and thus the maximum velocity occurs at this overshoot and is consequently slightly larger than predicted by the approximate analytical model. For channel heights below $10 \mu \mathrm{m}$, the assumptions of a boundary-driven plug flow with a superimposed Poiseuille backflow begin to collapse as the height of the channel becomes comparable to the boundary-layer thickness, and a more elaborate analytical calculation of the streaming velocity field is necessary [12].

\section{Time-averaged second-order temperature}

In Fig. 5(a), we show the time-averaged second-order temperature field $T_{2}$ calculated for the default 380- $\mu \mathrm{m}$-by$160-\mu \mathrm{m}$ geometry at the resonance frequency. In Figs. 5(b) and 5(c), we show line plots of $T_{2}$ along the horizontal and vertical dashed lines in Fig. 5(a). $T_{2}$ has a saddle point in the center of the channel $(y=0, z=0)$, two global maxima on the horizontal centerline $z=0$, and a wide plateau on the vertical center line $y=0$. The temperature field is forced to be zero at all boundaries due to the boundary condition of infinite heat conduction. The gradient of $T_{2}$ along line $\mathrm{C}$ indicates a decline in heat generation inside the boundary layer going from the center toward the left and right walls. The global maxima in the bulk result from heat generation in the bulk as discussed in Sec. V.

\section{DISCUSSION}

In Fig. 6(a) we provide an overview of the energy transport and dissipation in the system by showing a sketch of the energy currents in the channel cross section. To explain the convection of energy, we consider the first-order velocity to be composed of a weak nonresonant part $\boldsymbol{v}_{1}^{\text {bc }}$, which fulfills the oscillating velocity boundary conditions, and a strong resonant part $\boldsymbol{v}_{1}^{\text {res }}$, which has zero amplitude at all walls [32]. In Fig. 6(b), we show the total energy current density, given by all the terms inside the divergence in Eq. (15c), in the bulk of the channel, thus not including the thin boundary layers at the top and bottom walls. The plot shows how mechanical energy is entering the system at the left and right walls, due to the
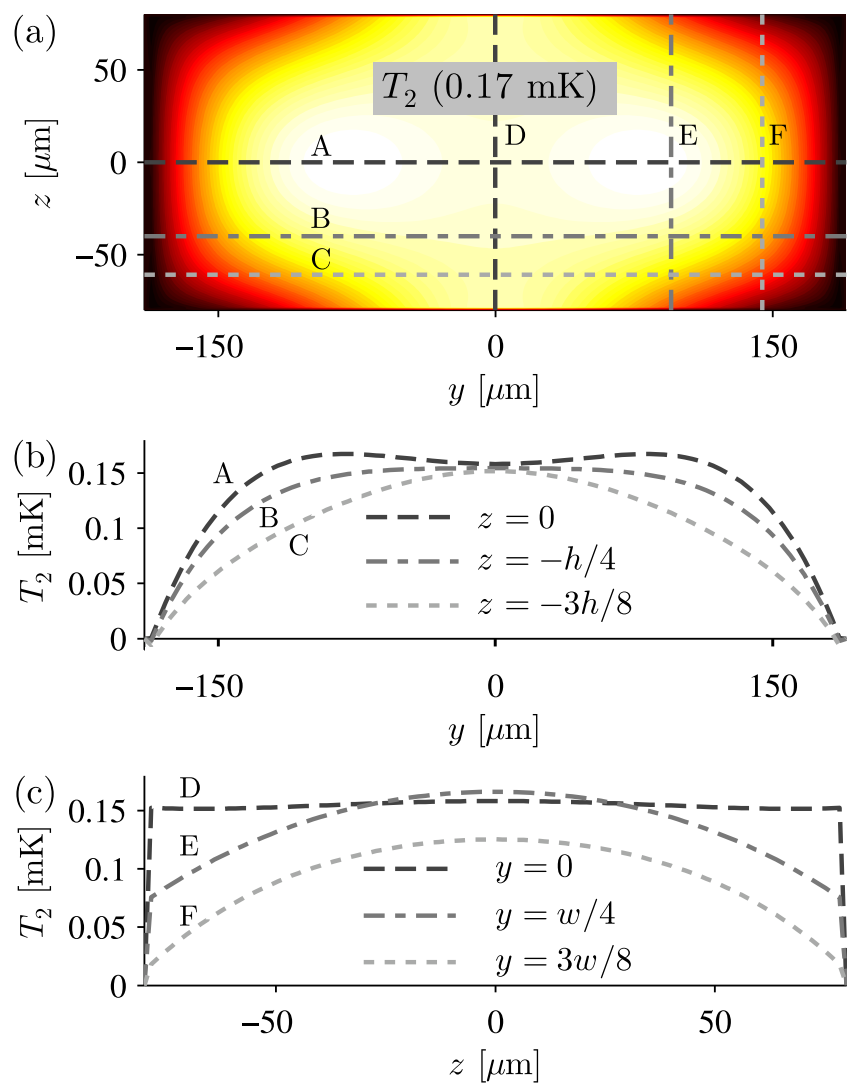

FIG. 5. (Color online) Time-averaged second-order temperature $T_{2}$ calculated for the default $380-\mu \mathrm{m}$-by-160- $\mu \mathrm{m}$ geometry, actuation frequency $f_{\text {res }}=1.9669923 \mathrm{MHz}$, and equilibrium temperature $T_{0}=25^{\circ}$ C. (a) Color plot (black $0 \mathrm{mK}$ to white $0.17 \mathrm{mK}$ ) of $T_{2}$ in the channel cross section. (b) and (c) Line plots of $T_{2}$ along the horizontal and vertical dashed lines in (a), respectively.

oscillating boundary condition, and is convected toward the top and bottom walls. This transport is dominated by the term $\left\langle p_{1} \boldsymbol{v}_{1}\right\rangle$ in Eq. (15c), particularly the nonresonant part $\left\langle p_{1} \boldsymbol{v}_{1}^{\mathrm{bc}}\right\rangle$, since $\boldsymbol{v}_{1}^{\text {res }}$ is out of phase with $p_{1}$ in the bulk. The $y$ and $z$ components of the energy current density inside the boundary layer at the bottom wall are shown in Figs. 6(c) and 6(d). The transport parallel to the wall, Fig. 6(c), results from $\left\langle p_{1} v_{1}^{\text {res }}\right\rangle$, which is large, since $\boldsymbol{v}_{1}^{\text {res }}$ is phase-shifted inside the boundary layer. The transport perpendicular to the wall, Fig. 6(d), results predominantly from the thermal diffusion term $-k_{0}^{\text {th }} \nabla T_{2}$.

To rationalize the amplitudes of the fields, we estimate the order of magnitude of the energy transport and dissipation in the system. The incoming energy current density from the oscillating velocity boundary condition at the left and right walls is given by the time-averaged product of the local pressure and velocity $\left\langle p_{1} v_{y 1}^{\mathrm{bc}}\right\rangle$. Multiplying this by the area $2 h \ell$, we obtain the magnitude of the incoming power $P_{\text {in }} \sim 2 h \ell \frac{1}{2} p_{1}^{\mathrm{a}} v_{\mathrm{bc}}$. Here, the factor $\frac{1}{2}$ enters from time averaging, $\ell$ is the channel length, and the superscript "a" denotes the amplitude of the resonant field. From the inviscid part of the first-order momentum conservation Eq. (9a), we estimate the magnitude $p_{1}^{\mathrm{a}} \sim \rho_{0} c_{s} v_{y 1}^{\mathrm{a}}$ and therefore obtain $P_{\text {in }} \sim h \ell \rho_{0} c_{s} v_{y 1}^{\mathrm{a}} v_{\mathrm{bc}}$. 

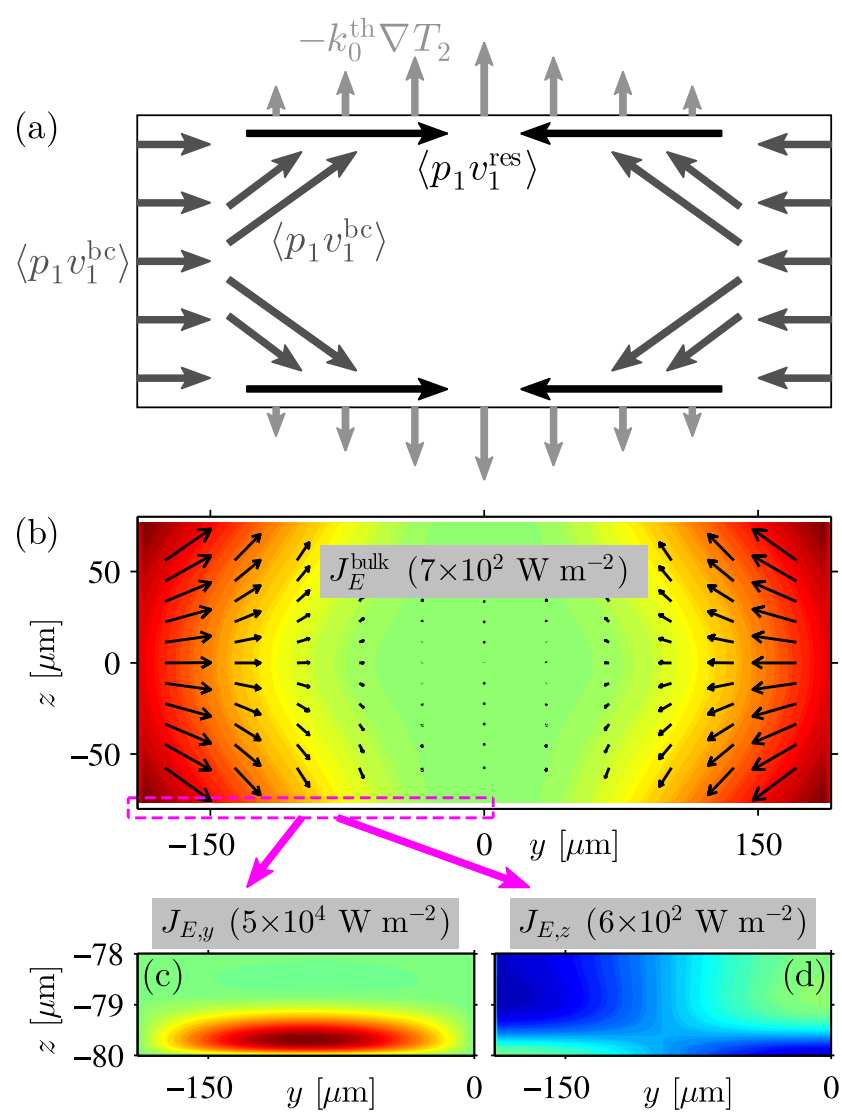

FIG. 6. (Color online) Time-averaged heat current densities in the channel cross section. (a) Sketch of the heat currents (arrows) with indication of the responsible terms in the time-averaged energy conservation Eq. (15). (b) Heat current density (arrows) and its magnitude [color plot ranging from zero (light green) to $7 \times 10^{2} \mathrm{~W}$ $\mathrm{m}^{-2}$ (dark red)] in the bulk of the channel. The strong currents inside the boundary layers are not shown. (c) Magnitude of the $y$ component of the heat current density [color plot ranging from zero (light green) to $5 \times 10^{4} \mathrm{~W} \mathrm{~m}^{-2}$ (dark red)] inside the boundary layer at the bottom wall. (d) Magnitude of the $z$ component of the heat current density [color plot ranging from $-6 \times 10^{2} \mathrm{~W} \mathrm{~m}^{-2}$ (dark blue) to zero (light green)] inside the boundary layer at the bottom wall.

The dissipation of mechanical energy happens primarily in the viscous boundary layers of thickness $\delta_{\mathrm{s}}$ due to the work done by the viscous stress force density $\left(\nabla \cdot \tau_{1}\right)$ with power density $\left\langle\left(\nabla \cdot \boldsymbol{\tau}_{1}\right) \cdot \boldsymbol{v}_{1}\right\rangle$. As the gradient of $\boldsymbol{v}_{1}$ perpendicular to the wall inside the boundary layer is large, the dominant term is $\left\langle\eta_{0} \frac{\partial^{2} v_{y 1}}{\partial z^{2}} v_{y 1}\right\rangle \sim \eta_{0} \frac{1}{4}\left(v_{y 1}^{\mathrm{a}}\right)^{2} / \delta_{\mathrm{s}}^{2}$, where two factors of $\frac{1}{2}$ enter from spatial and time averaging. The total power dissipation is given by the product of the power density and the volume of the boundary layers, $P_{\mathrm{s}} \sim 2 \delta_{\mathrm{s}} w \ell \eta_{0} \frac{1}{4}\left(v_{y 1}^{\mathrm{a}}\right)^{2} / \delta_{\mathrm{s}}^{2}$.

In steady state, $P_{\mathrm{s}}$ equals $P_{\text {in }}$, from which we find the magnitude $v_{y 1}^{\mathrm{a}}$ of the resonant field in terms of $v_{\mathrm{bc}}$ to be $v_{y 1}^{\mathrm{a}} \sim \frac{2}{\pi} \frac{h}{\delta_{\mathrm{s}}} \frac{\lambda}{w} v_{\mathrm{bc}} \sim 500 v_{\mathrm{bc}}$ for our system, which is in good agreement with the numerical result for $v_{y 1}^{\mathrm{a}} / v_{\mathrm{bc}}$ plotted in the inset of Fig. 3.

To rationalize the magnitude of the second-order temperature shift, we consider the diffusive energy transport through the top and bottom walls. The diffusive energy current density
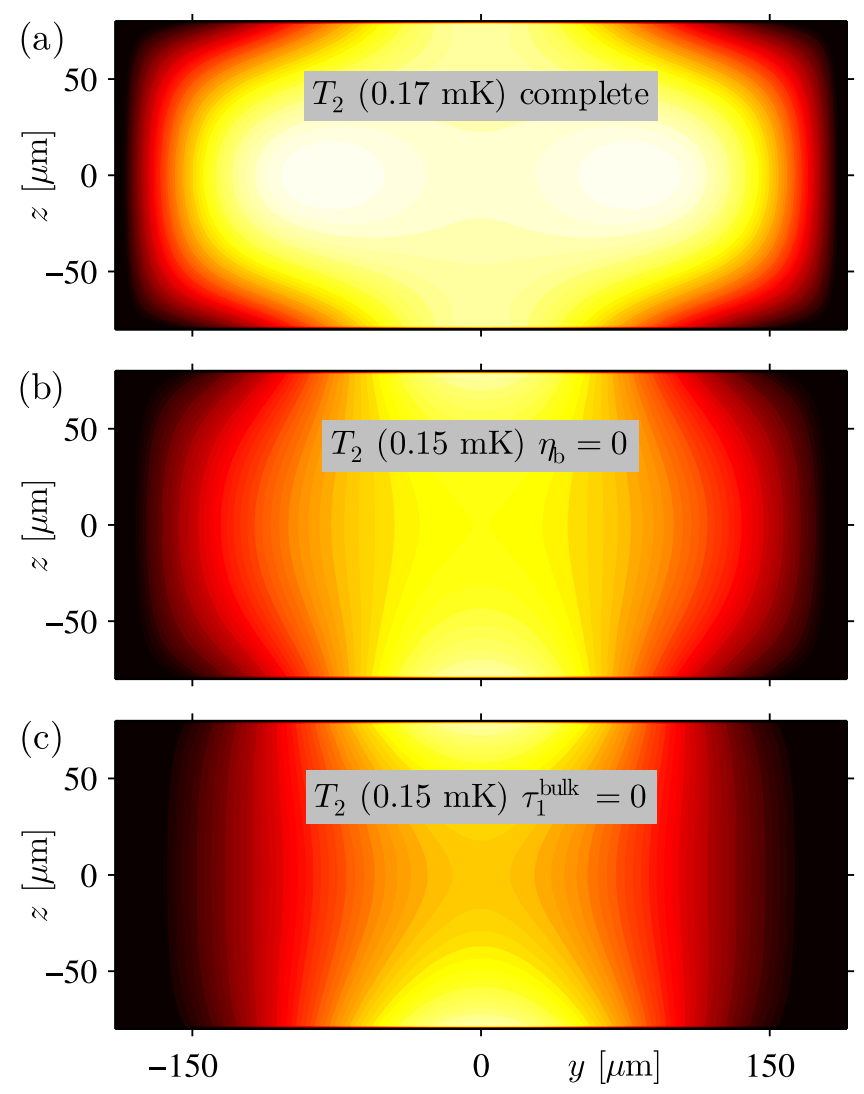

FIG. 7. (Color online) Color plot of the time-averaged secondorder temperature $T_{2}$ [from zero (black) to maximum (white)] in three cases. (a) $T_{2}$ calculated from the complete governing equations, identical to Fig. 5(a). (b) $T_{2}$ calculated without bulk viscosity $\eta^{\mathrm{b}}=0$. (c) $T_{2}$ calculated with zero viscous stress $\boldsymbol{\tau}=\mathbf{0}$ in the bulk defined by $|y|<(w / 2-4 \mu \mathrm{m})$ and $|z|<(h / 2-4 \mu \mathrm{m})$.

is $-k_{0}^{\text {th }} \nabla T_{2}$, and as heat diffuses to the perfectly conducting walls on a length scale of $\delta_{t}$, the outgoing power is $P_{\text {out }} \sim$ $2 w \ell k_{0}^{\text {th }}\left(\frac{1}{2} T_{2}^{\mathrm{a}} / \delta_{\mathrm{t}}\right)$. Here, the spatial average of $T_{2}$ just outside the thermal boundary layers along the top and bottom walls has been approximated to $\frac{1}{2} T_{2}^{\mathrm{a}}$. In steady state, $P_{\text {out }}$ equals $P_{\text {in }}$ and the magnitude of the second-order temperature becomes $T_{2}^{\mathrm{a}} \sim \frac{2}{\pi^{2}} \frac{h^{2}}{\delta_{\mathrm{t}} \delta_{\mathrm{s}}}\left(\frac{\lambda}{w}\right)^{2} \frac{1}{c_{p}}\left(v_{\mathrm{bc}}\right)^{2} \sim 0.13 \mathrm{mK}$, which is comparable to the numerical result in Fig. 5.

From the simplified picture of strong heat generation inside the boundary layers, it may seem odd that the second-order temperature field in Fig. 5 has two global maxima in the bulk of the channel. This effect is due to the absorption in the bulk of the channel originating from the nonzero divergence of the stress force term $\left\langle\boldsymbol{v}_{1} \cdot \boldsymbol{\tau}_{1}\right\rangle$ in Eq. (15c) as shown in Fig. 7. In Fig. 7(a), we show the complete second-order temperature field $T_{2}$. Fig. 7(b) shows an artificial temperature field calculated without bulk viscosity, $\eta^{\mathrm{b}}=0$. No maxima appear in the bulk, and the temperature field looks more as expected from the simplified view of heat generation in the boundary layers. However, there is still a small amount of heat generation in the bulk of the channel from the shear viscosity. In Fig. 7(c) this heat generation is suppressed by setting $\boldsymbol{\tau}_{1}=\mathbf{0}$ in the bulk more than $4 \mu \mathrm{m}$ from the walls, while maintaining the full $\boldsymbol{\tau}_{1}$ in the boundary layers. The resulting plot of $T_{2}$ shows how heat 
is generated in boundary layers near the top and bottom walls and mainly conducted out of these, while some of the heat is conducted into the bulk and out through the left and right walls. The bulk viscosity $\eta^{\mathrm{b}}$ is often neglected when working at frequencies around $2 \mathrm{MHz}$ because of its small contribution to the total dissipation, and the subsequent negligible influence on the resonance curve and the streaming velocity field. However, Fig. 7 clearly shows that the bulk absorption is important for the spatial structure of the time-averaged temperature field.

In Sec. II B we stated that the changes in the dynamic viscosity due to its temperature and density dependence are $0.33 \%$ and $0.37 \%$, respectively, for the amplitudes of the acoustic oscillation used in this paper. It might seem surprising that, first, such a small perturbation of the viscosity can increase the magnitude of the streaming by $19 \%$ at $25^{\circ} \mathrm{C}$ as stated in Sec. IV B (39\% at $\left.50^{\circ} \mathrm{C}\right)$, and secondly, the numerical results are in very good agreement with the analytical expression from Ref. [15], which does not include the density dependence of the dynamic viscosity of similar magnitude as the temperature dependence. The explanation lies within the spatial structure of the fields. From the timeaveraged momentum Eq. (14), we see that the divergence of the stress tensor leads to a term containing the gradient of the viscosity perturbation $\left\langle\nabla \eta_{1} \cdot\left[\nabla \boldsymbol{v}_{1}+\left(\nabla \boldsymbol{v}_{1}\right)^{\mathrm{T}}\right]\right\rangle$. Here, $\eta_{1}$ is proportional to $T_{1}$ and $\rho_{1}$, and since $T_{1}$ changes on the small length scale of the boundary layer, whereas $\rho_{1}$ only changes on the long length scales of the channel width, we get $\nabla \eta_{1}=\nabla \eta_{1}^{(T)}+\nabla \eta_{1}^{(\rho)} \sim \eta_{1}^{(T)} / \delta_{\mathrm{t}}+\eta_{1}^{(\rho)} / w \approx \eta_{1}^{(T)} / \delta_{\mathrm{t}}$, where the superscripts refer to the contribution from either the temperature or the density perturbation. Consequently, with respect to the acoustic streaming, the temperature dependence of the dynamic viscosity is much more important than the density dependence.

The significant increase of the acoustic streaming magnitude, due to the temperature-induced viscosity perturbation, influences the interplay between radiation forces and drag forces on suspended particles [20,33]. The steady temperature rise of less than $1 \mathrm{mK}$, on the other hand, has a negligible influence on acoustic handling of biological samples, however other applications of acoustofluidics, such as thermoacoustic engines, rely on the steady energy currents for pumping heat from a low-temperature source to a high-temperature sink, or inversely, for generating acoustic power from the heat flow between a high-temperature source and a low-temperature sink [34,35].

\section{CONCLUSION}

In this work, we have presented a full numerical study of the acoustic streaming in the cross section of a long straight microchannel including the temperature and density dependence of the fluid viscosity and thermal conductivity. The temperature dependence of the streaming amplitude in the case of a deep microchannel agreed well with the analytical single-wall result from 2011 by Rednikov and Sadhal [15], whereas significant deviations were found for shallow channels. This strong dependence of the streaming amplitude on the channel height was explained qualitatively with a simple one-dimensional backflow model.
Furthermore, we showed that a meaningful comparison of solutions at different temperatures and off-resonance frequencies could be performed by normalizing the secondorder velocity field to the square of the first-order velocity amplitude.

We have also solved the time-averaged second-order energy conservation equation numerically and calculated the steady temperature rise in the channel, and we analyzed the energy transport in the system. For acoustophoretic devices, the temperature rise is less than $1 \mathrm{mK}$ and has no consequences for either operation conditions or biological samples. However, in other applications such as thermoacoustic engines, the energy transport is important.

Finally, we have provided polynomial fits in the temperature range from 10 to $50^{\circ} \mathrm{C}$ of the thermodynamic properties and transport properties of water at ambient atmospheric pressure based on data from IAPWS, which cover a much wider range of temperatures and pressures. This allows for easy implementation of the official parameter values for the properties of water in other models working under the same temperature and pressure conditions.

With the inclusion of the local perturbation in viscosity and thermal conductivity, due to their temperature and density dependence, we have solved the complete time-averaged secondorder acoustic equations for a Newtonian fluid enclosed by vibrating walls, with the one exception of the unknown density dependence of the bulk viscosity. To further progress the numerical analysis of microchannel acoustic streaming, one should improve the modeling of the vibration of the walls, preferably including the elastic waves in the surrounding solid material. In the present model, the acoustic streaming velocity field depends strongly on the choice of actuation conditions on the walls.

\section{ACKNOWLEDGMENTS}

We thank Wolfgang Wagner, Ruhr-Universität Bochum, for providing us with the software FLUIDCAL, Version Water (IAPWS-95), for calculating the thermodynamic properties of water. This work was supported by the Danish Council for Independent Research (DFF): Technology and Production Sciences (Grant No. 11-107021).

\section{APPENDIX A: IAPWS FORMULATION}

To ease the use of the official IAPWS values for the thermodynamic and transport properties of water in our numerical analysis, we fit polynomials in temperature to the data. The precise fitting procedure and its validation are described in the following.

The data for the thermodynamic properties are obtained from an Excel implementation [36] of the IAPWS Formulation 1995 [24], in which the equation of state for water is fitted using a function with 56 parameters covering the range $T_{\text {melt }} \leqslant$ $T \leqslant 1273.15 \mathrm{~K}$ and $p \leqslant 1000 \mathrm{MPa}$. The shear viscosity is taken from the IAPWS Formulation 2008 [25], and the thermal conductivity is taken from the IAPWS Formulation 2011 [26], for which we have implemented the expressions stated in the papers to extract data values in the temperature and density range of interest to us. The data for the density derivatives of 

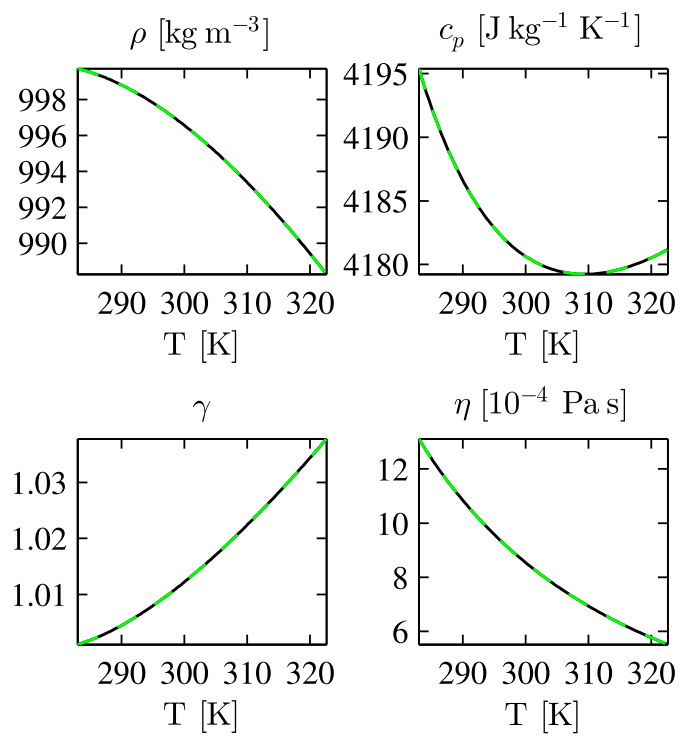
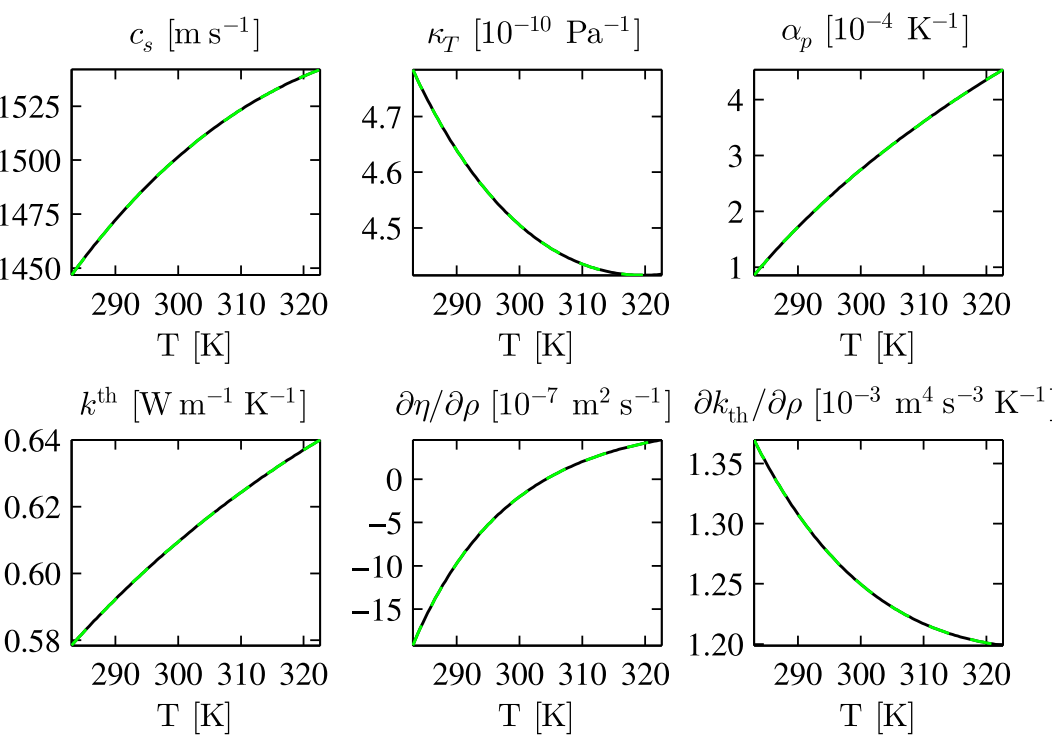

FIG. 8. (Color online) Fifth-order polynomial fits (dashed light green lines) to IAPWS data [24-26] (full black lines) of ten thermodynamic and transport parameters of water in the temperature range from $283 \mathrm{~K}\left(10^{\circ} \mathrm{C}\right)$ to $323 \mathrm{~K}\left(50{ }^{\circ} \mathrm{C}\right)$.

the viscosity and the thermal conductivity have been obtained using a central difference $\partial \eta / \partial \rho \approx[\eta(T, \rho+d \rho)-\eta(T, \rho-$ $d \rho)] /(2 d \rho)$, with $d \rho=0.001 \mathrm{~kg} \mathrm{~m}^{-3}$. The bulk viscosity is taken from Holmes, Parker, and Povey [27], who extended the work by Dukhin and Goetz [28]. The former paper provides a third-order polynomial fit in temperature to $\eta^{\mathrm{b}}$, thus rendering further fitting superfluous.

For each parameter, we extract 400 data points equally spaced in the temperature range from 283 to $323 \mathrm{~K}$ at ambient pressure $p=0.101325 \mathrm{MPa}$. From these 400 values, we only use every fourth point for the fitting of a fifth-order polynomial, while the remaining 300 data points are used for calculating the deviation of the fit from the data. The order of the polynomial has been chosen as a tradeoff between low deviation between fit and data, achieved at high polynomial order, and low uncertainty in the polynomial coefficients, achieved at low polynomial order. The fitted polynomial coefficients are then truncated to a finite precision of 11 significant digits. The number of significant digits has been chosen such that the finite precision of the fitting coefficients does not result in larger deviations between fit and data. We then calculated the relative deviation of the fit with respect

TABLE III. List of the coefficients $C_{i}$ of the fifth-order polynomial fits to the IAPWS data for water [24-26], shown in Fig. 8. The temperature dependence of each of the ten thermodynamic or transport parameters $g$ with SI unit $[g]$ is fitted in the temperature range from $283 \mathrm{~K}\left(10^{\circ} \mathrm{C}\right)$ to $323 \mathrm{~K}\left(50^{\circ} \mathrm{C}\right)$ by a polynomial of the form $g(\tilde{T}) /[\mathrm{g}]=C_{0}+C_{1} \tilde{T}+C_{2} \tilde{T}^{2}+C_{3} \tilde{T}^{3}+C_{4} \tilde{T}^{4}+C_{5} \tilde{T}^{5}$, with $\tilde{T}=T /(1 \mathrm{~K})$. MD and $\mathrm{AD}$ denote the maximum and average relative deviation, respectively, of the fit from the data. Text files with the polynomial coefficients are provided in the Supplemental Material [37].

\begin{tabular}{|c|c|c|c|c|c|}
\hline & $\rho$ & $c_{p}$ & $c_{s}$ & $\kappa_{T}$ & $\alpha_{p}$ \\
\hline$C_{0}$ & $-8.8657129122 \times 10^{+3}$ & $2.9011416353 \times 10^{+5}$ & $-3.1420310908 \times 10^{+4}$ & $1.4812304299 \times 10^{-7}$ & $-6.2142868017 \times 10^{-1}$ \\
\hline$C_{1}$ & $1.4835428749 \times 10^{+2}$ & $-4.4547002259 \times 10^{+3}$ & $4.4207693183 \times 10^{+2}$ & $-2.2647220701 \times 10^{-9}$ & $9.6088951712 \times 10^{-3}$ \\
\hline$C_{2}$ & $-8.9906362105 \times 10^{-1}$ & $2.7826280608 \times 10^{+1}$ & $-2.4555531077 \times 10^{+0}$ & $1.3993170593 \times 10^{-11}$ & $-5.9809671543 \times 10^{-5}$ \\
\hline$C_{3}$ & $2.7498750299 \times 10^{-3}$ & $-8.7096216461 \times 10^{-2}$ & $7.0411177557 \times 10^{-3}$ & $-4.3493756936 \times 10^{-14}$ & $1.8708110371 \times 10^{-7}$ \\
\hline$C_{4}$ & $-4.2441616455 \times 10^{-6}$ & $1.3656418313 \times 10^{-4}$ & $-1.0353739103 \times 10^{-5}$ & $6.7914632960 \times 10^{-17}$ & $-2.9366903765 \times 10^{-10}$ \\
\hline$C_{5}$ & $2.6348942330 \times 10^{-9}$ & $-8.5786317870 \times 10^{-8}$ & $6.1949139692 \times 10^{-9}$ & $-4.2558992023 \times 10^{-20}$ & $1.8495258230 \times 10^{-13}$ \\
\hline MD & $0.1 \times 10^{-6}$ & $1.2 \times 10^{-6}$ & $0.2 \times 10^{-6}$ & $5.3 \times 10^{-6}$ & $1.244 \times 10^{-4}$ \\
\hline \multirow[t]{2}{*}{$\mathrm{AD}$} & $0.0 \times 10^{-6}$ & $0.3 \times 10^{-6}$ & $0.1 \times 10^{-6}$ & $1.4 \times 10^{-6}$ & $0.119 \times 10^{-4}$ \\
\hline & $\frac{\gamma}{\gamma}$ & $\begin{array}{c}\eta \\
\end{array}$ & $k^{\text {th }}$ & $\partial \eta / \partial \rho$ & $\partial k_{\mathrm{th}} / \partial \rho$ \\
\hline$C_{0}$ & $8.6095341563 \times 10^{+1}$ & $3.8568288635 \times 10^{+0}$ & $-4.5378052364 \times 10^{+1}$ & $-3.7043919290 \times 10^{-2}$ & $3.3029688424 \times 10^{-1}$ \\
\hline$C_{1}$ & $-1.3297233662 \times 10^{+0}$ & $-6.0269041999 \times 10^{-2}$ & $7.0158464759 \times 10^{-1}$ & $5.8520864113 \times 10^{-4}$ & $-4.6998644326 \times 10^{-3}$ \\
\hline$C_{2}$ & $8.3329451753 \times 10^{-3}$ & $3.7823493660 \times 10^{-4}$ & $-4.3372914723 \times 10^{-3}$ & $-3.7051700834 \times 10^{-6}$ & $2.7065291331 \times 10^{-5}$ \\
\hline$C_{3}$ & $-2.6194143444 \times 10^{-5}$ & $-1.1905791749 \times 10^{-6}$ & $1.3522238266 \times 10^{-5}$ & $1.1749472916 \times 10^{-8}$ & $-7.8584696013 \times 10^{-8}$ \\
\hline$C_{4}$ & $4.1304638557 \times 10^{-8}$ & $1.8785807901 \times 10^{-9}$ & $-2.1181858883 \times 10^{-8}$ & $-1.8657255651 \times 10^{-11}$ & $1.1504432763 \times 10^{-10}$ \\
\hline$C_{5}$ & $-2.6113941825 \times 10^{-11}$ & $-1.1881783857 \times 10^{-12}$ & $1.3309059289 \times 10^{-11}$ & $1.1866022487 \times 10^{-14}$ & $-6.7916212133 \times 10^{-14}$ \\
\hline MD & $1.6 \times 10^{-6}$ & $1.379 \times 10^{-4}$ & $1.0 \times 10^{-6}$ & $15.459 \times 10^{-4}$ & $0.8 \times 10^{-6}$ \\
\hline $\mathrm{AD}$ & $0.4 \times 10^{-6}$ & $0.227 \times 10^{-4}$ & $0.3 \times 10^{-6}$ & $3.851 \times 10^{-4}$ & $0.1 \times 10^{-6}$ \\
\hline
\end{tabular}


to each of the 400 data points and derive the maximum relative deviation (MD) and the average relative deviation (AD). The derivative of the shear viscosity with respect to the density is a special case since it crosses zero in the temperature interval, and thus the deviation between the fit and the data points is normalized to the mean of all the data points instead of the local data point. The data points and the polynomial fits for all fitted parameters are shown in Fig. 8, and the polynomial coefficients and the maximum and average relative deviation between fit and data are shown in Table III. The fitted coefficients are provided in the Supplemental Material [37].
[1] H. Bruus, J. Dual, J. Hawkes, M. Hill, T. Laurell, J. Nilsson, S. Radel, S. Sadhal, and M. Wiklund, Lab Chip 11, 3579 (2011).

[2] M. Nordin and T. Laurell, Lab Chip 12, 4610 (2012).

[3] P. Thevoz, J. D. Adams, H. Shea, H. Bruus, and H. T. Soh, Anal. Chem. 82, 3094 (2010).

[4] M. Ohlin, A. E. Christakou, T. Frisk, B. Onfelt, and M. Wiklund, J. Micromech. Microeng. 23, 035008 (2013).

[5] J. Shi, D. Ahmed, X. Mao, S.-C. S. Lin, A. Lawit, and T. J. Huang, Lab Chip 9, 2890 (2009).

[6] A. Bussonniere, Y. Miron, M. Baudoin, O. Bou Matar, M. Grandbois, P. Charette, and A. Renaudin, Lab Chip 14, 3556 (2014).

[7] P. Augustsson, C. Magnusson, M. Nordin, H. Lilja, and T. Laurell, Anal. Chem. 84, 7954 (2012).

[8] T. Schwarz, G. Petit-Pierre, and J. Dual, J. Acoust. Soc. Am. 133, 1260 (2013).

[9] L. Rayleigh, Philos. Trans. R. Soc. London 175, 1 (1884).

[10] H. Schlichting, Phys. Z. 33, 327 (1932).

[11] W. L. Nyborg, J. Acoust. Soc. Am. 30, 329 (1958).

[12] M. Hamilton, Y. Ilinskii, and E. Zabolotskaya, J. Acoust. Soc. Am. 113, 153 (2003).

[13] M. Hamilton, Y. Ilinskii, and E. Zabolotskaya, J. Acoust. Soc. Am. 114, 3092 (2003).

[14] P. B. Muller, M. Rossi, Á. G. Marin, R. Barnkob, P. Augustsson, T. Laurell, C. J. Kähler, and H. Bruus, Phys. Rev. E 88, 023006 (2013).

[15] A. Y. Rednikov and S. S. Sadhal, J. Fluid Mech. 667, 426 (2011).

[16] W. L. Nyborg, J. Acoust. Soc. Am. 25, 68 (1953).

[17] C. Lee and T. Wang, J. Acoust. Soc. Am. 85, 1081 (1989).

[18] J. Lei, P. Glynne-Jones, and M. Hill, Lab Chip 13, 2133 (2013).

[19] J. Lei, M. Hill, and P. Glynne-Jones, Lab Chip 14, 532 (2014).

[20] P. B. Muller, R. Barnkob, M. J. H. Jensen, and H. Bruus, Lab Chip 12, 4617 (2012).

[21] M. Antfolk, P. B. Muller, P. Augustsson, H. Bruus, and T. Laurell, Lab Chip 14, 2791 (2014).
[22] L. D. Landau and E. M. Lifshitz, Statistical Physics, Part 1, 3rd ed. (Butterworth-Heinemann, Oxford, 1980), Vol. 5.

[23] A. D. Pierce, Acoustics (Acoustical Society of America, Woodbury, NY, 1991).

[24] W. Wagner and A. Pruss, J. Phys. Chem. Ref. Data 31, 387 (2002).

[25] M. L. Huber, R. A. Perkins, A. Laesecke, D. G. Friend, J. V. Sengers, M. J. Assael, I. N. Metaxa, E. Vogel, R. Mares, and K. Miyagawa, J. Phys. Chem. Ref. Data 38, 101 (2009).

[26] M. L. Huber, R. A. Perkins, D. G. Friend, J. V. Sengers, M. J. Assael, I. N. Metaxa, K. Miyagawa, R. Hellmann, and E. Vogel, J. Phys. Chem. Ref. Data 41, 033102 (2012).

[27] M. J. Holmes, N. G. Parker, and M. J. W. Povey, J. Phys.: Conf. Ser. 269, 012011 (2011).

[28] A. S. Dukhin and P. J. Goetz, J. Chem. Phys. 130, 124519 (2009).

[29] H. Bruus, Theoretical Microfluidics (Oxford University Press, Oxford, 2008).

[30] P. Augustsson, R. Barnkob, S. T. Wereley, H. Bruus, and T. Laurell, Lab Chip 11, 4152 (2011).

[31] COMSOL MULTIPHYSICS 4.4, www.comsol.com (2013).

[32] H. Bruus, Lab Chip 12, 20 (2012).

[33] R. Barnkob, P. Augustsson, T. Laurell, and H. Bruus, Phys. Rev. E 86, 056307 (2012).

[34] N. Rott, Z. Angew. Math. Phys. 26, 43 (1975).

[35] G. Swift, J. Acoust. Soc. Am. 84, 1145 (1988).

[36] U. Overhoff and W. Wagner, Fluidcal, version water (iapws-95), https://www.thermo.rub.de/en/prof-wwagner/software/fluidcal.html (2013).

[37] See Supplemental Material at http://link.aps.org/supplemental/ 10.1103/PhysRevE.90.043016 for text files with the fitted polynomial coefficients for the temperature dependence of the thermodynamic and transport parameters, both in a general format coefficients_general_format.txt for copy-paste use, and a format coefficients_comsol_format.txt for direct import into COMSOL MULTIPHYSICS. 